\title{
Sum rules of four-neutrino mixing in matter
}

\author{
He Zhang \\ CCAST (World Laboratory), P.O. Box 8730, Beijing 100080, China \\ and Institute of High Energy Physics, Chinese Academy of Sciences, \\ P.O. Box 918 (4), Beijing 100049, China* \\ (Electronic address: zhanghe@mail.ihep.ac.cn)
}

\begin{abstract}
Assuming the existence of one light sterile neutrino, we investigate the neutrino flavor mixing matrix in matter. Sum rules between the mixing parameters in vacuum and their counterparts in matter are derived. By using these new sum rules, we obtain the simple but exact expressions of the effective flavor mixing matrix in matter in terms of neutrino masses and the mixing parameters in vacuum. The rephasing invariants, sides of unitarity quadrangles and oscillation probabilities in matter are also achieved. Our model-independent results will be very helpful for analyzing flavor mixing and $\mathrm{CP}$ violation in the future long-baseline neutrino oscillation experiments.
\end{abstract}

${ }^{*}$ Mailing address 


\section{INTRODUCTION}

The facts that neutrinos are massive and lepton flavors are mixed have been confirmed by recent solar [1], atmospheric [2], reactor [3] and accelerator [4] neutrino oscillation experiments. However, the data coming from the Liquid Scintillation Neutrino Detector (LSND) experiment [5] have neither been confirmed nor refuted by the MiniBooNe experiment [6]. If the LSND result is proved to be true, most probably there exist more than three neutrino mass eigenstates. From the phenomenological point of view, the most straightforward and attractive way to understand the result of LSND is to introduce the sterile neutrinos, which are electroweak singlets and do not couple to the Standard Model $W$ and $Z$ bosons. Such sterile neutrinos mix with the active neutrinos, thus they can significantly influence neutrino oscillations. The sterile neutrinos may also be the candidates of warm dark matter [8] in cosmology. Due to the Mikheyev, Smirnov and Wolfenstein (MSW) effect [9] within the supernovae, visible effects of sterile neutrinos in supernova explosion will probably be detected in the forthcoming neutrino telescopes [10]. There are also plenty of other reasons to believe the possible existence of sterile neutrinos [11]. Even if the LSND experiment is not confirmed, sterile neutrinos can not be fully excluded, and the number of neutrino species is still an important open question in neutrino physics.

The direct effect of introducing sterile neutrinos is the mixing between active neutrinos

and sterile neutrinos. For simplicity, here we only consider one light sterile neutrino. Hence the neutrino mass eigenstates will become linear combinations of three active neutrinos $\left(\nu_{e}, \nu_{\mu}, \nu_{\tau}\right)$ and one sterile neutrino $\left(\nu_{s}\right)$. To be explicit, we can write

$$
\left(\begin{array}{c}
\nu_{s} \\
\nu_{e} \\
\nu_{\mu} \\
\nu_{\tau}
\end{array}\right)=\left(\begin{array}{cccc}
V_{s 0} & V_{s 1} & V_{s 2} & V_{s 3} \\
V_{e 0} & V_{e 1} & V_{e 2} & V_{e 3} \\
V_{\mu 0} & V_{\mu 1} & V_{\mu 2} & V_{\mu 3} \\
V_{\tau 0} & V_{\tau 1} & V_{\tau 2} & V_{\tau 3}
\end{array}\right)\left(\begin{array}{c}
\nu_{0} \\
\nu_{1} \\
\nu_{2} \\
\nu_{3}
\end{array}\right)
$$

where $\nu_{i}(i=0,1,2,3)$ denote the neutrino mass eigenstates, and $V_{\alpha i}(\alpha=s, e, \mu, \tau)$ is the lepton flavor mixing matrix.

The behavior of neutrino propagation in vacuum can be described by the effective Hamiltonian

$$
\mathcal{H}=\frac{1}{2 E}\left(V D^{2} V^{\dagger}\right)
$$

where $D \equiv \operatorname{Diag}\left\{m_{0}, m_{1}, m_{2}, m_{3}\right\}, m_{i}$ are the masses of $\nu_{i}$ and $E$ denotes the neutrino beam energy. In order to measure the lepton flavor mixing angles precisely and to answer the question whether neutrinos violate $\mathrm{CP}$, future accelerator experiments with very long baselines [12] are needed. In such long baseline experiments, neutrino beams should travel through the terrestrial matter, and their propagation can be significantly modified when they interact with matter. The effective Hamiltonian responsible for neutrinos travelling in matter can be written in a form analogous to Eq. (2)

$$
\tilde{\mathcal{H}}=\frac{1}{2 E}\left(\tilde{V} \tilde{D}^{2} \tilde{V}^{\dagger}\right)
$$


where $\tilde{D} \equiv \operatorname{Diag}\left\{\tilde{m}_{0}, \tilde{m}_{1}, \tilde{m}_{2}, \tilde{m}_{3}\right\}, \tilde{m}_{i}$ are the effective neutrino masses in matter and $\tilde{V}$ denotes the effective lepton mixing matrix in matter. $\tilde{\mathcal{H}}$ is related with $\mathcal{H}$ as

$$
\tilde{\mathcal{H}}-\mathcal{H}=\frac{1}{2 E} \mathcal{A}
$$

where $\mathcal{A}=2 E \times \operatorname{Diag}\left(a^{\prime}, a, 0,0\right)$ with the matter parameters $a^{\prime}=\sqrt{2} G_{\mathrm{F}} N_{n} / 2$ and $a=$ $\sqrt{2} G_{\mathrm{F}} N_{e}$ arising from coherent forward neutrino scattering [13]. Here $G_{\mathrm{F}}$ is the Fermi constant, $N_{e}$ and $N_{n}$ are the numbers of electrons and neutrons per unit volume. For most realistic long-baseline neutrino experiments, the background density can usually be treated as a constant [14] $\left(N_{e}=\right.$ constant and $N_{n}=$ constant). In this work, we take $N_{e}$ and $N_{n}$ as constants.

In respect that the phenomenology of neutrino oscillations in matter can be formulated in terms of $\tilde{V}$ and $\tilde{m}_{i}$, a number of authors have discussed the analytical relations between $\tilde{V}$ and $V$ [15-22] in the framework of three active neutrinos. The main purpose of this paper is to derive the exact analytical relationships between $\tilde{V}$ and $V$ in the four-neutrino mixing scheme. After an extension of our previous results [21] from three active neutrinos to three active neutrinos and one sterile neutrino, the model-independent sum rules between $\left(\tilde{V}, \tilde{m}_{i}\right)$ and $\left(V, m_{i}\right)$ are given in section II. The relations between moduli $\left|\tilde{V}_{\alpha i}\right|$ and $\left|V_{\alpha i}\right|$ are then obtained in section III, and so are the relations between $\tilde{V}_{\alpha i} \tilde{V}_{\beta i}^{*}$ and $V_{\alpha i} V_{\beta i}^{*}$. Applications of our relations are also shown in section III. Finally, a brief summary is presented in section IV.

\section{SUM RULES IN THE FOUR-NEUTRINO MIXING SCHEME}

The effective Hamiltonians $\mathcal{H}$ and $\tilde{\mathcal{H}}$ in Eqs. (2) and (3) can be expressed as

$$
\begin{aligned}
\mathcal{H}_{\alpha \beta} & =\frac{1}{2 E} \sum_{i} m_{i}^{2} V_{\alpha i} V_{\beta i}^{*}, \\
\tilde{\mathcal{H}}_{\alpha \beta} & =\frac{1}{2 E} \sum_{i} \tilde{m}_{i}^{2} \tilde{V}_{\alpha i} \tilde{V}_{\beta i}^{*},
\end{aligned}
$$

where the Greek indices $\alpha, \beta$ and so on generally run over $s, e, \mu$ and $\tau$, and the Latin indices $i, j$, etc. run over $0,1,2$ and 3 here after. Eq. (4) turns out to be

$$
\sum_{i} \tilde{m}_{i}^{2} \tilde{V}_{\alpha i} \tilde{V}_{\beta i}^{*}=\sum_{i} m_{i}^{2} V_{\alpha i} V_{\beta i}^{*}+\mathcal{A}_{\alpha \beta}
$$

with

$$
\mathcal{A}_{\alpha \beta}=a^{\prime} \delta_{s \alpha} \delta_{s \beta}+a \delta_{e \alpha} \delta_{e \beta}
$$

Here only the $(s, s)$ and $(e, e)$ elements of $\mathcal{A}$ are not vanishing. The unitarity conditions of $V$ and $\tilde{V}$ yield

$$
\sum_{i} V_{\alpha i} V_{\beta i}^{*}=\sum_{i} \tilde{V}_{\alpha i} \tilde{V}_{\beta i}^{*}=\delta_{\alpha \beta} .
$$


These two sets of sum rules are straightforward and obvious, but they are not enough for our calculations. In order to obtain the relations between $\tilde{V}_{\alpha i} \tilde{V}_{\beta i}^{*}$ and $V_{\alpha i} V_{\beta i}^{*}$, we need more linear independent equations.

Taking into account the unitarity of $V(\tilde{V})$, we find that $(\mathcal{H})^{n}=(1 / 2 E)^{n} V D^{2 n} V^{\dagger}$ and $(\tilde{\mathcal{H}})^{n}=(1 / 2 E)^{n} \tilde{V} \tilde{D}^{2 n} \tilde{V}^{\dagger}$ hold, in which $n=0, \pm 1, \pm 2$, etc. To be explicit, we obtain the elements of $\mathcal{H}(\tilde{\mathcal{H}})$ as

$$
\begin{aligned}
\left(\mathcal{H}^{n}\right)_{\alpha \beta} & =\frac{1}{(2 E)^{n}} \sum_{i} m_{i}^{2 n} V_{\alpha i} V_{\beta i}^{*}, \\
\left(\tilde{\mathcal{H}}^{n}\right)_{\alpha \beta} & =\frac{1}{(2 E)^{n}} \sum_{i} \tilde{m}_{i}^{2 n} \tilde{V}_{\alpha i} \tilde{V}_{\beta i}^{*} .
\end{aligned}
$$

Note that the case $n=0$ corresponds to the unitarity conditions, and $n=1$ corresponds to the sum rules in Eq. (6). For the case $n=2$, we square the two sides of Eq. (6) and obtain

$$
\sum_{i} \tilde{m}_{i}^{4} \tilde{V}_{\alpha i} \tilde{V}_{\beta i}^{*}=\sum_{i}\left[m_{i}^{4}+m_{i}^{2}\left(\mathcal{A}_{\alpha \alpha}+\mathcal{A}_{\beta \beta}\right)\right] V_{\alpha i} V_{\beta i}^{*}+\mathcal{A}_{\alpha \beta}^{2} .
$$

In the calculation of Eq. (10), the expression of $\mathcal{A}$ in Eq. (7) has been considered. For the case $n=3$, we get the forth set of equations by cubing both two sides of Eq. (6)

$$
\begin{aligned}
\sum_{i} \tilde{m}_{i}^{6} \tilde{V}_{\alpha i} \tilde{V}_{\beta i}^{*}= & \sum_{i}\left[m_{i}^{6}+m_{i}^{4}\left(\mathcal{A}_{\alpha \alpha}+\mathcal{A}_{\beta \beta}\right)+m_{i}^{2}\left(\mathcal{A}_{\alpha \alpha}^{2}+\mathcal{A}_{\beta \beta}^{2}+\mathcal{A}_{\alpha \alpha} \mathcal{A}_{\beta \beta}\right)\right] V_{\alpha i} V_{\beta i}^{*} \\
& +\sum_{\gamma, i, j} m_{i}^{4} V_{\alpha i} V_{\gamma j} V_{\gamma i}^{*} V_{\beta j}^{*} \mathcal{A}_{\gamma \gamma}+\mathcal{A}_{\alpha \beta}^{3} .
\end{aligned}
$$

Eqs. (6), (8), (10) and (11) constitute a full set of linear equations of $\tilde{V}_{\alpha i} \tilde{V}_{\beta i}^{*}$. By solving these equations, one can derive the exact expressions of $\tilde{V}_{\alpha i} \tilde{V}_{\beta i}^{*}$ in terms of $\tilde{m}_{i}, m_{i}$ and $V_{\alpha i} V_{\beta i}^{*}$. Note that, the sum rules obtained above are only valid for neutrinos. As for antineutrinos, the corresponding sum rules can directly be obtained by replacing $(V, \tilde{V}, \mathcal{A})$ with $\left(V^{*}, \tilde{V}^{*}\right.$, $-\mathcal{A}$ ). Our formulae can be generalized to include more than one sterile neutrinos. In this paper, we only concentrate on the four-neutrino mixing scheme and the main results will be given in the next section.

\section{THE MIXING MATRIX ELEMENTS IN MATTER}

The sum rules obtained in the previous section totally contain ten sets of linear equations, which are related to the ten independent elements in $\tilde{\mathcal{H}}$. Let us first consider the case $\alpha=\beta$, in which the sum rules are simplified to

$$
\begin{aligned}
\sum_{i}\left|\tilde{V}_{\alpha i}\right|^{2} & =\sum_{i}\left|V_{\alpha i}\right|^{2}=1, \\
\sum_{i} \tilde{m}_{i}^{2}\left|\tilde{V}_{\alpha i}\right|^{2} & =\sum_{i} m_{i}^{2}\left|V_{\alpha i}\right|^{2}+\mathcal{A}_{\alpha \alpha}, \\
\sum_{i} \tilde{m}_{i}^{4}\left|\tilde{V}_{\alpha i}\right|^{2} & =\sum_{i}\left[m_{i}^{4}+2 m_{i}^{2} \mathcal{A}_{\alpha \alpha}\right]\left|V_{\alpha i}\right|^{2}+\mathcal{A}_{\alpha \alpha}^{2},
\end{aligned}
$$




$$
\begin{aligned}
\sum_{i} \tilde{m}_{i}^{6}\left|\tilde{V}_{\alpha i}\right|^{2}= & \sum_{i}\left[m_{i}^{6}+2 m_{i}^{4} \mathcal{A}_{\alpha \alpha}+3 m_{i}^{2} \mathcal{A}_{\alpha \alpha}^{2}\right]\left|V_{\alpha i}\right|^{2} \\
& +\sum_{\gamma, i, j} m_{i}^{4} V_{\alpha i} V_{\gamma j} V_{\gamma i}^{*} V_{\alpha j}^{*} \mathcal{A}_{\gamma \gamma}+\mathcal{A}_{\alpha \alpha}^{3} .
\end{aligned}
$$

After a lengthy calculation, the solutions of Eq. (12) are obtained as

$$
\left|\tilde{V}_{\alpha i}\right|^{2}=\frac{1}{\prod_{k \neq i} \tilde{\Delta}_{i k}} \sum_{j}\left(F_{\alpha}^{i j}+C_{\alpha}\right)\left|V_{\alpha j}\right|^{2},
$$

where

$$
\begin{aligned}
F_{\alpha}^{i j} & =\prod_{k \neq i}\left(\mathcal{A}_{\alpha \alpha}+\widehat{\Delta}_{j k}\right) \\
C_{\alpha} & =-\frac{1}{2} \sum_{\gamma, m, n} \Delta_{m n}^{2} V_{\alpha m} V_{\gamma n} V_{\gamma m}^{*} V_{\alpha n}^{*} \mathcal{A}_{\gamma \gamma} .
\end{aligned}
$$

Here we have defined the mass-squared differences $\Delta_{i j} \equiv m_{i}^{2}-m_{j}^{2}, \tilde{\Delta}_{i j} \equiv \tilde{m}_{i}^{2}-\tilde{m}_{j}^{2}$ and $\widehat{\Delta}_{i j} \equiv m_{i}^{2}-\tilde{m}_{j}^{2}$. In deriving Eq. (13), we have taken into account the relationship [23]

$$
\sum_{i} \tilde{m}_{i}^{2}=\sum_{i} m_{i}^{2}+\sum_{\alpha} \mathcal{A}_{\alpha \alpha}
$$

which can be clearly seen from the traces of two sides of Eq. (4). Note that only the mass-squared differences appear in our results, and the terms in proportion to the absolute neutrino masses disappear. This is consistent with the fact that oscillation processes are irrelevant to the absolute neutrino masses. Eq. (13) is very instructive and compact since it clearly shows the connections between $\left|V_{\alpha i}\right|$ and $\left|\tilde{V}_{\alpha i}\right|$. The survival probabilities denoted by $P\left(\nu_{\alpha} \rightarrow \nu_{\alpha}\right)$ or $P\left(\bar{\nu}_{\alpha} \rightarrow \bar{\nu}_{\alpha}\right)$ [23] are only sensitive to the moduli of $V(\tilde{V})$ and thus these expressions will be very helpful for phenomenological analyses of the disappearance processes in the neutrino oscillation experiments.

For the case $\alpha \neq \beta$, we obtain the explicit expressions

$$
\tilde{V}_{\alpha i} \tilde{V}_{\beta i}^{*}=\frac{1}{\prod_{k \neq i} \tilde{\Delta}_{i k}}\left(\sum_{j} F_{\alpha \beta}^{i j} V_{\alpha j} V_{\beta j}^{*}+C_{\alpha \beta}\right),
$$

where

$$
\begin{aligned}
F_{\alpha \beta}^{i j} & =\frac{1}{2}\left[-\widehat{\Delta}_{j i}\left(\mathcal{A}_{\alpha \alpha}^{2}+\mathcal{A}_{\beta \beta}^{2}+4 \mathcal{A}_{\alpha \alpha} \mathcal{A}_{\beta \beta}\right)+\prod_{k \neq i}\left(\mathcal{A}_{\alpha \alpha}+\mathcal{A}_{\beta \beta}+\widehat{\Delta}_{j k}\right)+\prod_{k \neq i} \widehat{\Delta}_{j k}\right], \\
C_{\alpha \beta} & =-\frac{1}{2} \sum_{\gamma, m, n} \Delta_{m n}^{2} V_{\alpha m} V_{\gamma n} V_{\gamma m}^{*} V_{\beta n}^{*} \mathcal{A}_{\gamma \gamma},
\end{aligned}
$$

and $\alpha \neq \beta$. In the limit $\mathcal{A} \rightarrow 0$, our results are reduced to the vacuum case $\tilde{V} \rightarrow V$.

To show the applications of our results, we define the rephasing invariants in matter as 


$$
\tilde{J}_{\alpha \beta}^{i j} \equiv \operatorname{Im}\left(\tilde{V}_{\alpha i} \tilde{V}_{\beta j} \tilde{V}_{\alpha j}^{*} \tilde{V}_{\beta i}^{*}\right)
$$

which are similar to the one in the three-neutrino mixing scheme [24]. Only nine of these invariants are independent [25]. We can choose any set of nine independent $\tilde{J}_{\alpha \beta}^{i j}$ to fully describe $\mathrm{CP}$ and $\mathrm{T}$ violation in matter.

The twelve unitarity quadrangles [25], which are the geometrical presentations of the orthogonality of $V(\tilde{V})$ in the complex plane, are well related with $\mathrm{CP}$ and $\mathrm{T}$ violation in neutrino oscillations. The twenty-four sides of six quadrangles have been given in Eq. (16). Once precise measurements are provided in future, we can systematically analyze the matter effects on these quadrangle sides by taking use of our relations.

The probability of one neutrino $\nu_{\alpha}$ converting to another neutrino $\nu_{\beta}$ in matter is given as

$$
P\left(\nu_{\alpha} \rightarrow \nu_{\beta}\right)=\delta_{\alpha \beta}-4 \sum_{i<j}\left[\operatorname{Re}\left(\tilde{V}_{\alpha i} \tilde{V}_{\beta j} \tilde{V}_{\alpha j}^{*} \tilde{V}_{\beta i}^{*}\right) \sin ^{2} \tilde{F}_{j i}\right]-2 \sum_{i<j}\left(\tilde{J}_{\alpha \beta}^{i j} \sin 2 \tilde{F}_{j i}\right)
$$

where $\tilde{F}_{j i} \equiv 1.27 \tilde{\Delta}_{j i} L / E$ with $L$ standing for the baseline length (in unit of km) and $E$ being the neutrino beam energy (in unit of $\mathrm{GeV}$ ). From our simple and exact formulae, the oscillation probabilities for all channels in matter can be calculated and the $\mathrm{CP}$-violating information can also be extracted.

It should be mentioned that our formulae are also very suitable for numerical analyses, and the relevant work will be elaborated elsewhere.

\section{SUMMARY}

Motivated by the interesting extension of the standard flavor mixing scheme of three active neutrinos with one sterile neutrino, we have derived a full set of sum rules in the fourneutrino mixing scheme. By using these sum rules, we have calculated the effective neutrino mixing matrix in matter $\tilde{V}$ in terms of $\tilde{m}_{i}, m_{i}$ and the genuine neutrino mixing matrix $V$. The rephasing invariants, sides of six unitarity quadrangles and oscillation probabilities in matter can be obtained by using our formulae in Eqs. (13) and (16). These compact and simple results are expected to be very helpful to describe the leptonic flavor mixing and $\mathrm{CP}$ and $\mathrm{T}$ violation in matter in a model-independent way. The relations are also very useful for a systematic study of neutrino oscillations in the future long-baseline experiments.

The author would like to thank Professor Z.Z. Xing for reading the manuscript, making many corrections and giving a number of helpful suggestions. He is also grateful to S. Zhou and W.L. Guo for useful discussions. This work was supported in part by the National Nature Science Foundation of China. 


\section{REFERENCES}

[1] SNO Collaboration, Q.R. Ahmad et al., Phys. Rev. Lett. 87 (2001) 071301; 89 (2002) 011301; 89 (2002) 011302.

[2] Super-Kamiokande Collaboration, Y. Fukuda et al., Phys. Lett. B 467 (1999) 185; S. Fukuda et al., Phys. Rev. Lett. 85 (2000) 3999; Phys. Rev. Lett. 86 (2001) 5656.

[3] KamLAND Collaboration, K. Eguchi et al., Phys. Rev. Lett. 90 (2003) 021802.

[4] K2K Collaboration, M.H. Ahn et al., Phys. Rev. Lett. 90 (2003) 041801.

[5] LSND collaboration, Phys. Rev. Lett. 77 (1996) 3082.

[6] The MiniBooNE Collaboration (E. Church et al.) FERMILAB-P-0898 (1997), available at http://library.fnal.gov/archive/test-proposal/0000/fermilab-proposal-0898.shtml.

[7] D. Caldwell and R. N. Mohapatra, Phys. Rev. D 46 (1993) 3259; J. Peltoniemi and J. W. F. Valle, Nucl. Phys. B 406 (1993) 409; J. Peltoniemi, D. Tommasini and J. W. F. Valle, Phys. Lett. B 298 (1993) 383.

[8] S. Dodelson and L.M. Widrow, Phys. Rev. Lett. 72 (1994) 17; A.D. Dolgov, Phys. Rept. 370 (2002) 333.

[9] L. Wolfenstein, Phys. Rev. D 17 (1978) 2369; S.P. Mikheyev and A.Yu. Smirnov, Yad. Fiz. Sov. J. Nucl. Phys. 42 (1985) 1441.

[10] G. Dutta et al., Phys. Rev. D 62 (2000) 093014; Phys. Rev. D 64 (2001) 073011; M. Sorel and J.M. Conrad, Phys. Rev. D 66 (2002) 033009; J. Fetter et al., Astropart. Phys. 18 (2003) 433.

[11] R.N. Mohapatra et al., hep-ph/0510213.

[12] P. Fisher, B. Kayser, and K.S. McFarland, Ann. Rev. Nucl. Part. Sci. 49 (1999) 481; B. Autin et al., CERN 99-02 (1999); D. Ayres et al., physics/9911009; C. Albright et al., hep-ex/0008064; and references therein.

[13] See, e.g., S.M. Bilenky, C. Giunti, and W. Grimus, Prog. Part. Nucl. Phys. 43 (1999) 1 ; and references therein.

[14] I. Mocioiu and R. Shrock, Phys. Rev. D 62 (2000) 053017; and references therein.

[15] V. Barger, K. Whisnant, S. Pakvasa, and R.J. Phillips, Phys. Rev. D 22 (1980) 2718.

[16] H.W. Zaglauer and K.H. Schwarzer, Z. Phys. C 40 (1988) 273.

[17] Z.Z. Xing, Phys. Lett. B 487 (2000) 327; Phys. Rev. D 63 (2001) 073012; Phys. Rev. D 64 (2001) 073014.

[18] M. Freund, Phys.Rev.D 64 (2001) 053003.

[19] K. Kimura, A. Takamura, and H. Yokomakura, Phys. Lett. B 537 (2002) 86; Phys. Rev. D 66 (2002) 073005; Nucl. Phys. Proc. Suppl. 149 (2005) 191.

[20] Y. Farzan and A.Yu. Smirnov, Phys. Rev. D 65 (2002) 113001; E.Kh. Akhmedov, M. Maltoni and A.Yu. Smirnov, hep-ph/0506064.

[21] H. Zhang and Z.Z. Xing, Eur. Phys. J. C 41 (2005) 143; Z.Z. Xing and H. Zhang, Phys. Lett. B 618 (2005) 131.

[22] M. Honda, Y. Kao, N. Okamura and T. Takeuchi, hep-ph/0602115; P.F. Harrison and W.G. Scott, Phys. Lett. B 535 (2002) 229.

[23] Z.Z. Xing, Int. J. Mod. Phys. A 19 (2004) 1.

[24] C. Jarlskog, Phys. Rev. Lett. 55 (1985) 1039.

[25] W.L. Guo and Z.Z. Xing, Phys. Rev. D 65 (2002) 073020; Phys. Rev. D 66 (2002) 097302. 